\title{
INTELLIGENT INFORMATION SYSTEMS, QUO VADIS?
}

\author{
Vic Matta, Ohio University, matta@ ohio.edu \\ Dusan Sormaz, Ohio University, sormaz@ohio.edu
}

\begin{abstract}
Based on its most popular incarnations, Intelligent Information Systems (IIS) appears to be a subdiscipline of artificial intelligence with elements of Information Systems (IS). In asking Quo Vadis, (Latin for "whither are you going?"), we appear to engage in evolutionism. However, this article does not attempt to predict the entire evolutionary pattern of the subject. It merely contemplates the effects of continuation of currently observable trends in IS [6] and AI towards the growth of an academic subdiscipline of collective interest called Intelligent Information Systems. The goal is to understand and introduce some conceptual order into the phenomenon of proliferation of IS and AI types.
\end{abstract}

Keywords: Intelligent Information Systems, Information Systems, Artificial Intelligence, Scope and Definitions of Disciplines, Emerging Discipline

\section{INTRODUCTION}

Appropriate classification and organization of disciplines are conditional to a discussion on their development and progression. We discuss the current evolution of two well established and large disciplines involving information processing: artificial intelligence (AI) and information systems (IS). We outline constructs and evolution with the intention of plotting the locus of IIS. Both AI \& IS have been around for a long time but gained recognition in the second half of the twentieth century.

The first formal deductive reasoning system could be traced back to the $5^{\text {th }}$ Century B.C. when Aristotle invented syllogistic logic. However, John McCarthy [2] coined the term "artificial intelligence" in 1956 at the Darthmouth Conference. The American Association of Artificial Intelligence summarizes artificial intelligence as computational understanding of mechanisms underlying thought and intelligent behavior, their embodiment in machines and development of artifacts that utilize those methods.

Characterized by fundamental forms of technology used to solve the input processing, output and communication problems of its time, the first basic era of Information Systems began with the
Sumerians' cuneiform in Mesopotamia in 3000 B.C. [14]. As described by Laudon, et al. [14], we are in the fourth generation of digital computing, where computers as well as smart mobile devices are used by technical as well as non-technical people for business as well as personal uses. Communication has become an integral part of this age of diversifying technology.

We begin by reviewing classifications of these two disciplines in research and texts. We don't claim the sources of our references to be authoritarians in their field, and the review is by no means a survey, but more of a glimpse. Controversies already exist for such disciplines that develop around quickly evolving technology. As information technology changes, organizations are impacted in how they implement the new technology tools. This implementation underlies business processes that also need to adapt to new challenges. AI and IS are impacted by technology, changes, processes and research that is triggered by this implementation. The introduction of each definable system was followed by stream of research on its impact (e.g., Expert System (ES), Management Information Systems (MIS), Neural Networks (NN), etc. [6]). The objective of this paper is to understand the diversity of the AI \& IS disciplines and explain their organization. We then propose to use this discussion as a framework to provide some perspectives on the role and realm of Intelligent Information Systems (IIS).

\section{EXPLORING THE SCOPE OF IS AND AI DISCIPLINES}

In order to plot the space occupied by IIS, we must first express the framework composed by the interval between AI and IS, within which IIS will lie. In this section, we discuss the descriptives of IS and AI, and then plot where IIS is seen to have research and application.

\section{The Discipline of Information Systems}

Ein-Dor and Segev broadly define IS as any computerized system with a user or operator interface, provided that the computer is not physically embedded [6]. Broad definitions are suitable with disciplines that are less mature. 
According to Whinston and Geng, "A gray area exists when determining the research relevance issue of any academic discipline, because no discipline is bounded absolutely" [20]. They argue that IS still has a larger gray area than a benchmark mature discipline, based on the relatively larger number of associated issues. For example, the call for papers at the International Association for Computer Information Systems (IACIS) invites papers from 30 different topics that range from management oriented topics such as team building to more technological ones such as security or data warehousing.

Baskerville and Myers [1] argue that the IS discipline has completed its evolution and is becoming a "reference discipline." They categorize the areas of IS into management processes (e.g., strategic planning for infrastructure), developmental processes (e.g., IS project management and systems implementation), development concepts (e.g., social construction for requirements), its representations (e.g., database and knowledge base concepts) and applications (e.g., e-commerce systems).

\section{The Discipline of Artificial Intelligence}

According to Luger [15], the scope of AI initially appeared to be divided between an "analysis of formal theories of intelligence" and that "intelligence was some sort of grand hack that could be approached in an application driven, ad-hoc manner." Contemporary AI appears to have many more debates between logicians and designers of artificial life forms that evolve illogically, between designers of physical symbol systems and those of neural networks, between proponents of expert systems and case-based reasoning.

As with IS, there are many definitions of Artificial Intelligence, each stemming from narrower applications. Broadly speaking, Artificial Intelligence is a science of representation and reasoning [9] toward the use of machines to solve problems otherwise solved by intelligent beings [17].

Luger [15] defines AI as "the branch of computer science that is concerned with the automation of intelligent behavior." He suggests that AI is based on theoretical and applied doctrines of computer science, which include knowledge representation, and casebased reasoning-algorithms and programming needed to apply that knowledge. In this way, Burkholder argues that AI is an empirical science [3].

While there are several versions of definitions of AI, most experts agree that intelligent behavior and intelligence is central to AI. [4]. The problem of defining artificial intelligence then translates to one of defining intelligence and remains a difficult subject to define. What can be considered as evidence of intelligence? How are creativity, learning and selfawareness related to intelligence? Is computer-based intelligence viable, or must it be purely biological? These questions still have debatable answers. AI facilitates the exploration of these questions [15]. In response to the ambiguity in defining $\mathrm{AI}$, we maintain Luger's definition of AI as discussed above.

\section{Applications in AI}

Uncertain and unpredictable business environments are causing organizations to turn to AI and Expert Systems (ES) to develop knowledge management systems for competence. Knowledge discovery-and mining of data from databases, and state-of-the-art research in data analysis and pattern recognition are used to predict future outcomes [16]. For example, banks use neural networks to make better sense of banks of data to improve asset management, trading, credit card fraud detection, and portfolio management [22]. Like most sciences, AI has several subdisciplines:

State Space Search and Game Playing involve techniques to explore alternatives in the problem domain or heuristics, and study of methods and rules of discovery and invention.

Automated Reasoning uses computer programs to assist in the process of drawing logical conclusion from facts [21]. In Theorem Proving, the problem description is represented in formal logic in a realm of well defined domain of rules, and problems are treated as theorems to be proved.

Expert Systems (ES) and Knowledge Management (KM): Based on automated reasoning, ES have been considered a derivative, in that they use computer programs to reason as humans and often use axioms similar to those of Decision Support Systems (DSS), an IS discipline [5]. KM is the systematic collection, transfer, security and management of information .

Natural Language Understanding and Semantics: The automation of understanding and generating human language has been a long-standing AI objective. Applications of this type are in use with automated phone answering services.

Planning: This may involve two areas: hierarchical problem solving and programming the ability to 
respond to and organize plans around dynamically changing environments.

Robotics is the science and technology of making a mechanical device perform tasks autonomously or under the control of a pre-programmed computer.

Machine Learning is a method for creating computer programs by the analysis of data sets. Neural networks are non-linear statistical data modeling tools and are often be considered to be a subset of machine learning.

\section{INTELLIGENT INFORMATION SYSTEMS}

Perusal of industry and research sources shows increasing evidence of IIS. We have appraised some of these resources to look for similarities and differences in the classification of IIS. It is interesting to note that each of these instances of IIS have some variations in this taxonomy. As we will see in the examples that follow, the technology industry puts IIS closer to AI and engineering applications on the IS - AI continuum. The research and academic worlds, however, include DB in the classification. Also significant is that the formal research in IIS appears to have begun in 2000, when increasing digitization of information and Moore's law had revolutionized information creation and accessibility [8]. We see this as an indication of beginnings of a new field in response to the needs of applications of AI that involve IS.

The Intelligent Information Systems Institute (IISI) began operation in December of 2000 and is located in the Computing and Information Science department of Cornell University. Its primary objective is "to perform and stimulate research in compute- and data-intensive methods for intelligent decision making systems" [10]. The institute suggests IIS to be Computer Science and Operations Research oriented.

The Intelligent Information Systems Research Laboratory (IISRL) at Pennsylvania State University researches ways of enhancing the ability to generate, manage, search, and mine information and knowledge. They suggest the inclusion of database design and analysis, mobile web computing, web mining and navigation, web agents, novel and intelligent web tools, multimedia retrieval, web and internet models, web usage, automatic content analysis and digital libraries, web search, niche search engines, semantic web, scientific databases, data mining, and information retrieval [8]. This research lab presents IIS as a field of graduate research in web and database systems.

Laboratory for Intelligent Information Systems (IISLAB) belongs to the division of database and information techniques (ADIT) of Linköpings University in Sweden. IISLAB conducts research in advanced information management systems tailored to present and future information technology. Current projects focus on information security, information retrieval and filtering, and the representation, organization and processing of knowledge in distributed environments such as the World Wide Web [19]. In their opinion, IIS is IS with smart information processing.

Established in 1992, the Journal of Intelligent Information Systems (JIIS) describes IIS as the next generation of IS developed as a result of integration of AI and database technologies. According to the JIIS editors, IIS "embodies knowledge that allows it to exhibit intelligent behavior, cooperate with users and other systems in problem solving, discovery, access, recovery, retrieval, and manipulation of data and knowledge [12].

\section{Description of the IIS domain}

Figure 1 is a graphical representation of an Intelligent Information Systems expressed as an integration of IS (shown as the left most full circle) and AI (the right most full circle) with database technologies (DB) (the bottom center full circle). Knowledge Management is the area common to IS and AI circles, Database Management Systems (DBMS) is the area common to IS and Database (DB) technologies, and Knowledge Representation (KR) is the area common to $\mathrm{AI}$ and $\mathrm{DB}$. $\mathrm{KR}$ is underlying and unified representation of data and information within the subdisciplines of AI.

As shown in Figure 1, IIS is shown to be common to the three main areas: IS, AI and DB. The entities and activities supporting IIS (such as IISI, JIIS, IIRL and IISLAB) are marked with italicized text on gray backgrounds within the IIS area. JIIS and IISI see IIS as more AI and DB oriented than IISRL and IISLAB, which show evidence of more IS in IIS. This fact suggests that perhaps IIS is most influenced by AI, somewhat by DB, and IS. 


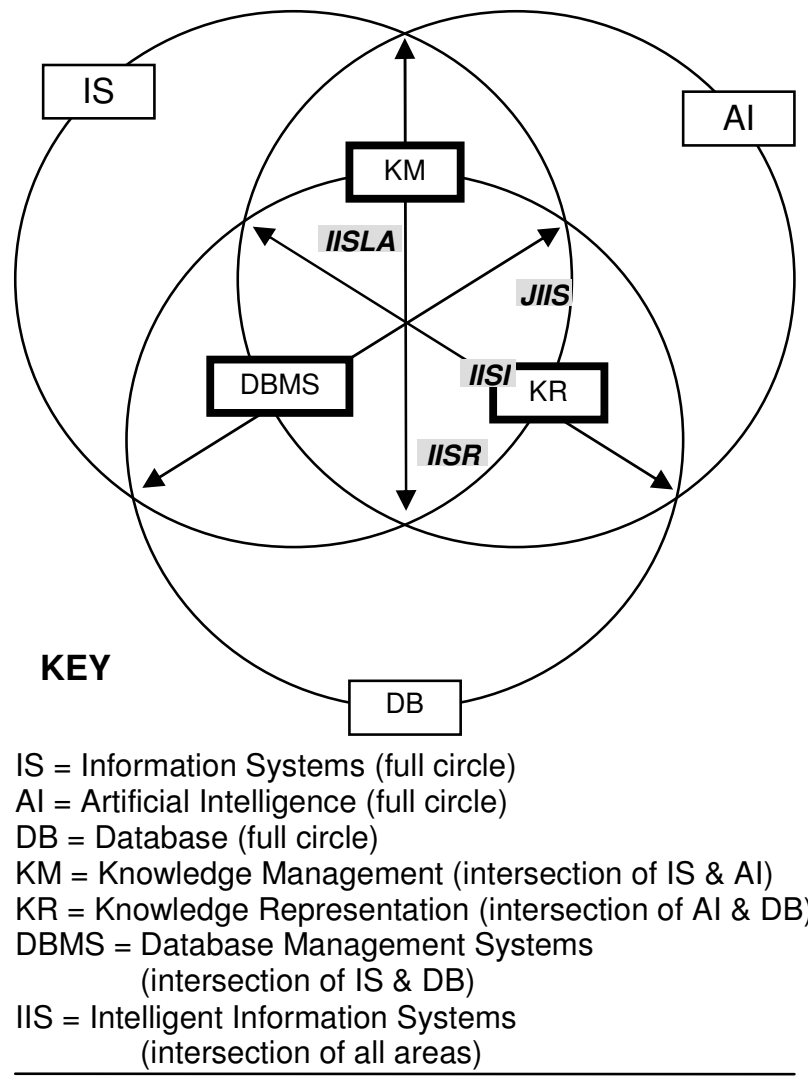

Figure 3. Domain of IIS

\section{Business Rules Management}

For businesses to make consistent decisions, their systems must be based on high quality logical rules and facts that are available to decision makers and decision support systems [11]. These rule-based systems are characterized by symbol-based learning methods in which a set of symbols represents the entities and relationships of a problem domain. For example, ID3 (Luger, 2005) is an algorithm that induces concepts from examples by representing concepts as decision trees that classify an object by testing values of its properties for information content. Consider the problem of estimating an individual's credit risk based on credit history, debt, collateral and income. The decision tree (Figure 2) can be built such that each node represents a test on one of the properties (such as debt, or collateral) with leaves corresponding to possible values of the target property (risk).

\section{Semantic Search Systems}

Kozichev [13] proposes that modern automated systems should include means for automating "semantic tasks" such as computer assisted translation semantic search, and processing of textual information. This intelligent application is demonstrated by Figure 3, where Google provides alternate spellings of the term(s) used for the search using Google's dictionary.

Regardless of the likelihood of the typographical error, Google still conducts the search (Figure 4). The IIS demonstrated here is one that uses ranking, which is based on semantic analysis.

Decision tree hierarchy for loan approval

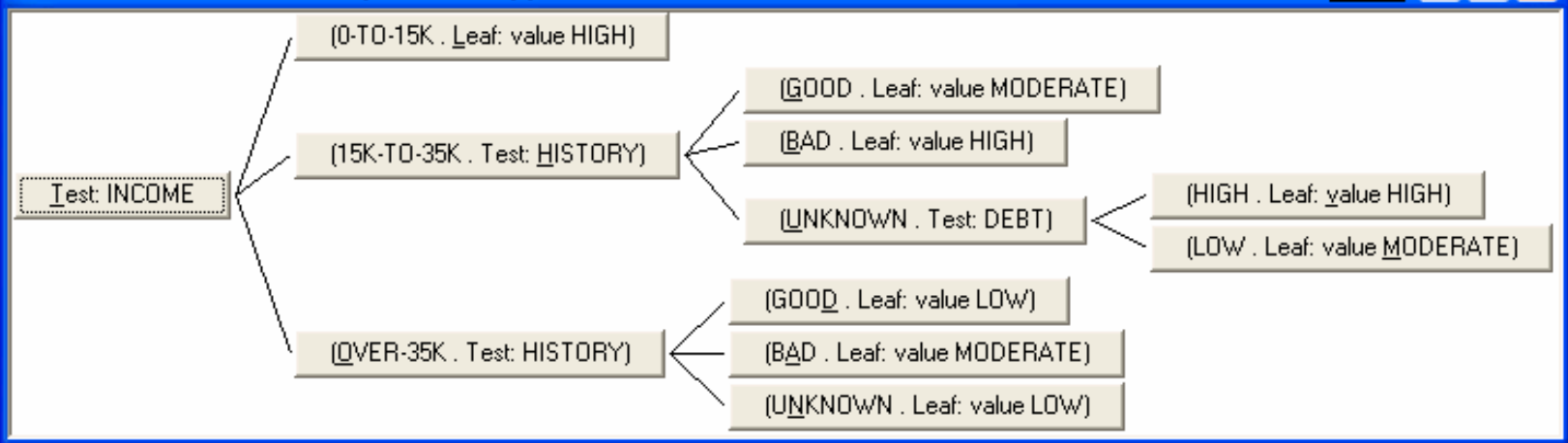

Figure 4. Business Rules Decision Tree 


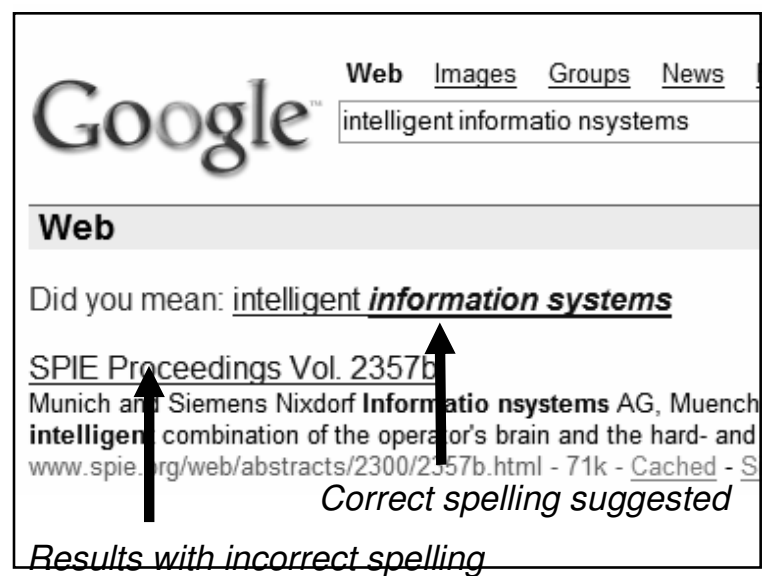

Figure 5. Google Suggests an Alternate Keyword Based on a Corrected Spelling

Web
Did you mean: planning
IT Planning Tools
$\begin{aligned} & \text { National Center for Technology Planing: School dis } \\ & \text { for Technology Planning: Buildings Level: Schools T } \\ & \text { ctc.aspira.org/planning.html - 17k - Cached - Simila }\end{aligned}$

Figure 6. Google Suggests an Alternate Keyword Based on Ranking

Engineering and technology-based applications of IIS are abundant. A typical application is described in [18], where computer-integrated manufacturing (CIM) is converted into an intelligent information system by combining CIM with concurrent engineering and knowledge management.. His requirements of IIS include the ability to access knowledge about previous products or designs instantly for consideration in reliability, maintainability, cost, quality, manufacturability, performance, etc. Low level automation tools should have the capability to notify and record design or manufacturing process changes.

\section{CONCLUSIONS}

JIIS published its first journal on IIS in 1992. IISRL, IISLAB and IISI have all emerged in 2000, reaffirming the existence of IIS as a sub-discipline. Based on the research and applications of the work done by these entities, it is clear that IIS occupies a space between IS, AI and DB. It could be argued that this space is not exactly in the middle, as indicated by the work carried out by these entities, but is closest to AI. For example, one of the most well established sources, the JIIS, summarizes IIS "as a result of integration of AI and database technologies."

IIS appears to have been spurred by diffusion of the ability to digitize information and the advent of the Internet, which allowed the ease of transfer of this information [6].

\section{Assumptions and Next Steps}

We assume that the entities surveyed to assess the existence of IIS are pioneers in the field, based on the people involved in these organizations and the fact that their work is contemporary and relevant.

The plotting of the location of the entities inspected in Figure 3 is suggestive only and is based on fields of research as listed by leading entities in IIS as of early 2006. It is likely that this would evolve [7].

A larger survey of existing research is needed to fully define the domain of IIS. Since the domains of parent disciplines (AI and IS) are changing, so is IIS. Relative newness of IIS contributes to a large "gray area" [20]. A study tracing emergence of applications of IIS since 2000 would be helpful in defining its realm.

\section{REFERENCES}

1. Baskerville, R. L., \& Myers, M. D. (2002). Information systems as a reference discipline. MIS Quarterly, 26(1), 1-14.

2. Buchanan, B. G. (2006). Brief history of artificial intelligence. Retrieved Feb 22, 2006, from http://www.aaai.org/AITopics/bbhist.html

3. Burkholder, L. (1999). Are AI and mechanics empirical disciplines? Journal of Experimental \& Theoretical Artificial Intelligence, 11(4), 497500.

4. Courtial, J.-P., \& Law, J. (1989). A co-word study of artificial intelligence. Social Studies of Science, 19, 301-311. 
5. Duan, Y., Edwards, J. S., \& Xuc, M. X. (2005). Web-based expert systems: Benefits and challenges. Information and Management, 42, 799-811.

6. Ein-Dor, P., \& Segev, E. (1993). A classification of information systems: Analysis and interpretation. Information Systems Research, 4(2), 166-204.

7. Galliers, R. D. (2004). Trans-disciplinary research in information systems. International Journal of Information Management, 24(1), pp. 99106.

8. Giles, L. (2000). Intelligent information systems research laboratory. Retrieved March12, 2006, 2006, from http://iis.ist.psu.edu/

9. Goebel, R. (1988). Distinguishing science and technology in artificial intelligence: A reply to d. Parnas. Information Systems and Operations Research, 26(4), 247-255.

10. Gomes, C. (2000, February 16, 2006). Intelligent information systems institute. Retrieved March 12, 2006, 2006, from http://www.cis.cornell.edu/iisi/

11. Halle, B. V. (2001). Business rules applied: Building better systems using the business rules approach (First Edition ed.): Wiley.

12. Kerschberg, L., Ras, Z. W., \& Zemankova, M. (1992). Journal of intelligent information systems. Retrieved March 12, 2006, 2006, from http://www.springerlink.com/link.asp?id=10028 9

13. Kozichev, C. V. N. (2004). Intelligent information systems: Application and principles of designing. Military Thought, 87-91.
14. Laudon, K. C., Traver, C. G., \& Laudon, J. P. (July 13, 1998). A history of information technology and systems. Information Technology and Systems, 2006, from http://www.tcf.ua.edu/AZ/ITHistoryOutline.htm

15. Luger, G. F. (2005). Artificial intelligence: Structures and strategies for problem solving: Pearson Education.

16. Mitchell, T. M. (1999). Machine learning and data mining. Comm. of the ACM, 42(11), 30-36.

17. Parnas, D. L. (1988). Why engineers should not use artificial intelligence. Information Systems and Operations Research, 26(4), 234-246.

18. Prasad, B. (2000). Converting computerintegrated manufacturing into an intelligent information system by combining cim with concurrent engineering and knowledge management. Industrial Management and Data Systems, 100(7), 301-316.

19. Shahmehri, N. (2006, 2005). Laboratory for intelligent information systems. Retrieved March 12, 2006, 2006

20. Whinston, A. B., \& Geng, X. (2004). Operationalizing the essential role of the information technology artifact in information systems research: Gray area, pitfalls, and the importance of strategic ambiguity. MIS Quarterly, 28(2), 149-159.

21. Wos, L. (1992). Automated reasoning: Introduction \& applications: McGraw Hill.

22. Young, K. (1999). A learning process. The Banker. 